\title{
La plage, les étoiles. Simon et l'Éros cosmique
}

\section{Giuseppe Zuccarino}

\section{OpenEdition}

\section{Journals}

Édition électronique

URL : http://journals.openedition.org/ccs/819

DOI : $10.4000 /$ ccs. 819

ISSN : 2558-782X

\section{Éditeur :}

Presses universitaires de Rennes, Association des lecteurs de Claude Simon

\section{Édition imprimée}

Date de publication : 31 décembre 2011

Pagination : 79-93

ISBN : 9782354121464

ISSN : 1774-9425

\section{Référence électronique}

Giuseppe Zuccarino, «La plage, les étoiles. Simon et l'Éros cosmique », Cahiers Claude Simon [En ligne] 7 | 2011, mis en ligne le 21 septembre 2017, consulté le 01 mai 2019. URL : http:// journals.openedition.org/ccs/819; DOI : 10.4000/ccs.819 


\section{La plage, les étoiles. Simon et l'Éros cosmique}

Giuseppe ZUCCARINO

Critique littéraire, Gênes

\section{L'apothéose de la chevelure}

Le roi égyptien Ptolémée III Evergète doit partir pour combattre dans la troisième guerre de Syrie (246-241 av. J.-C.). Sa jeune épouse, la reine Bérénice, promet de faire offrande aux dieux, dans le temple d'Arsinoé Zéphyritide, d'une natte de ses beaux cheveux si son mari est de retour indemne de la guerre. C'est une coutume déjà attestée par les poèmes d'Homère ${ }^{1}$, et qui dans le cas de Bérénice a une issue heureuse, puisque Ptolémée revient vainqueur. La reine tient sa promesse, mais quelques jours plus tard les cheveux disparaissent du temple. À ce moment-là, l'astronome de la cour, Conon de Samos, prétend avoir retrouvé la natte dans le ciel, en se référant à un groupe d'étoiles situées entre les constellations de la Vierge, du Lion et du Bouvier. L'effet du catastérisme (ce terme désigne le processus par lequel, dans la mythologie ancienne, un être humain ou

1. Dans l'Iliade, il est fait mention du fait que Pélée a promis que son fils Achille, s'il revenait vivant de Troie, offrirait au fleuve Sperchée sa propre chevelure. En réalité, Achille, conscient de devoir mourir loin de sa patrie, coupe ses cheveux pour les placer sur le bûcher des funérailles de son ami Patrocle. Voir Omero, Iliade, XXIII, Turin, Einaudi, 1984, p. 800-801. 
divin se transforme en étoile) est la naissance d'une nouvelle constellation, justement appelée Chevelure de Bérénice.

L'épisode est connu parce que Callimaque l'a choisi comme sujet d'un poème qui malheureusement nous est parvenu seulement dans une forme mutilée et fragmentaire. Dans ce poème, c'est la natte elle-même qui s'adresse à la reine, et lui rappelle, à regret, le moment où les cheveux ont étés sacrifiés ; ensuite, ils ont été soulevés par Zéphyr jusqu'au ciel et déposés sur les genoux d'Arsinoé (identifiée avec Aphrodite), laquelle a décidé de leur donner une nouvelle nature, celle d'étoiles².

Les nombreuses lacunes qui aujourd'hui appauvrissent le texte grec sont en partie compensées par la célèbre version que Catulle en a donnée dans l'un de ses poèmes ${ }^{3}$. Les vers du poète latin nous aident à suivre l'argumentation de la natte, qui ne manque pas de faire allusion à l'amour (y compris à l'attraction sexuelle) qui lie Bérénice à son époux et que l'on peut déduire de la douleur qu'elle éprouve lorsque Ptolémée doit partir à la guerre :

Quelles choses tristes tu disais, en prenant congé de ton époux !

Et combien de fois tu essuyais tes yeux de la main !

Quel dieu a été si puissant qu'il a pu changer ton caractère?

Ou bien les amants ne supportent pas de rester loin du corps qu'ils aiment ? ${ }^{4}$.

Le texte souligne ensuite que les cheveux coupés, au lieu de se sentir fiers de leur métamorphose en astres, regrettent leur emplacement d'origine dans la chevelure féminine. Enfin, la natte adresse à Bérénice une exhortation :

Toi, la reine, quand dans les jours de fête tu feras ton sacrifice

À la déesse Vénus, en levant tes yeux vers les étoiles,

Ne me laisse pas sans offrandes, mais honore-moi, puisque je suis

Une chose à toi, avec des offrandes très riches 5 .

2. Voir Callimaco, "La chioma di Berenice ", in Aitia, Giambi e altri frammenti, Milan, Rizzoli, 1996 ; 2007, p. 522-531.

3. Gaio Valerio Catullo, Le poesie, LXVI, Turin, Einaudi, 1997, p. 297-305.

4. Ibid., p. 300-301.

5. Ibid., p. 304-305. 
Donc, comme l'a remarqué un illustre traducteur et commentateur de ce texte, le poète Ugo Foscolo - l'un des créateurs du premier romantisme italien -, tout tend au même but : "Lautorité d'un astronome, les mérites et la passion de Bérénice, les victoires de Ptolémée rendent crédible l'apothéose de la chevelure sacrifiée $»^{6}$.

\section{D'un titre à l'autre}

Parmi les écrivains modernes, il est arrivé à Claude Simon d'évoquer, fût-ce indirectement, ce mythe. Il faut d'abord se rappeler qu'il n’a pas décidé immédiatement de donner à l'un de ses textes le titre La Chevelure de Bérénice. Celui-ci, dans sa première édition, il en avait un autre : Femmes'. Il s'agissait d'un livre d'art, de grand format et tiré à soixante exemplaires numérotés, qui comprenait, outre le texte de Simon, des bois gravés et les reproductions en couleurs de plusieurs tableaux de Joan Miró, ceux-ci se concentrant sur la représentation (mais certainement pas au sens réaliste du terme) de femmes. Les titres utilisés par le peintre étaient justement : Femme, Femme assise, Femme et oiseaus. Si Simon a accepté l'invitation, qui lui a été adressée par Aimé Maeght, d'accompagner d'un texte les œuvres de l'artiste espagnol, c'est pour plus d'une raison. Tout d'abord, à cause de son admiration pour Miró, dont il a même possédé un tableau?. Ensuite, parce que les images presque abstraites, purement allusives (basées sur la suggestion qui émane des épaisses lignes noires contrastant avec un fond plus neutre et d'autres zones de la toile peintes de couleurs vives), accordaient à Simon une grande liberté d'invention. Enfin, parce qu'il savait avoir en commun avec Miró des liens étroits avec l'Espagne, et en particulier avec la région de Barcelone.

6. Ugo Foscolo, "La chioma di Berenice " (1803), in Opere, I, Milan-Naples, Ricciardi, 1974, p. 273 ; voir aussi sa traduction en italien du poème de Catulle, p. 261-279.

7. Paris, Maeght, 1966 (mais le volume est « achevé d'imprimer le 27 novembre $1965 »)$.

8. Rappelons que Femme et oiseau est aussi le titre d'une photo prise par l'écrivain ; voir C. Simon, Photographies, 1937-1970, Paris, Maeght, 1992, p. 56-57.

9. L’espoir (1946), reproduit in Walter Erben, Joan Miró, Köln, Taschen, 1998, p. 118. 
Le lieu décrit dans Femmes est justement une plage catalane. Comme l'écrivain l'a expliqué, "au-delà du thème de la femme, le propre de cet ensemble de tableaux était d'évoquer des images d'une région que, comme Miró, je connais bien, la Catalogne, avec ses plages sur lesquelles Miró, comme moi, se promène, les yeux baissés, à la recherche du détritus jeté sur le sable par les vagues $»^{10}$. Le texte de Simon s'inspire des peintures de Miró, mais sans les commenter ni les décrire, puisqu'il se propose plutôt d'être une élaboration littéraire autonome. Les critiques ont cherché à trouver des similitudes entre certains passages du texte et les tableaux reproduits dans le livre ${ }^{11}$, mais ce qui semble encore plus important c'est l'analogie d'attitude entre le peintre et l'écrivain. Miró a déclaré : «Quant à mes moyens d'expression, je m'efforce [...] de provoquer d'abord une sensation physique, pour arriver ensuite à l'âme $»^{12}$. Et Simon semble lui faire écho lorsqu'il dit, à propos de l'un de ses romans, Le Palace (mais avec une formule qui convient très bien à un texte comme Femmes) : " J'ai voulu décrire des odeurs, des images, des sensations tactiles, des émotions $»^{13}$.

Il ne fait aucun doute cependant que les sujets des peintures, c'est-à-dire les figures de femmes et d'oiseaux (les unes et les autres transformées par l'artiste en idéogrammes personnalisés), sont très présentes dans le texte de Simon. Dans certains passages, même, elles sont mêlées les unes aux autres: l'on trouve par exemple une femme " criant comme un oiseau nocturne ", une "femme chouette " et d'autres qui ont des "yeux d'oiseaux fardés de bleu ou de vert $~^{114}$. Ces notations chromatiques nous rappellent qu'un autre des élé-

10. C. Simon, in Claude Du Verlie, "Claude Simon : the Crossing of the Image", in Diacritics, 4, 1977, p. 51 et in C. Simon, Euvres, Paris, Gallimard, 2006 (désormais E.), p. 1366. Dans Photographies, il y a plusieurs images de plages, bateaux, filets de pêche, détritus au bord de la mer.

11. Voir la " Notice " d'Alistair B. Duncan sur La Chevelure de Bérénice, in CE., p. $1362-1366$.

12. J. Miró, "Dichiarazione" (1933), in Lavoro come un giardiniere e altri scritti, Milan, Abscondita, 2008, p. 12.

13. C. Simon, in Madeleine Chapsal, "Entretien avec Claude Simon ", in L'Express, 5 avril 1962, p. 32 et in E., p. XXV-XXVI.

14. Pour les citations, nous nous référons à l'édition définitive, La Chevelure de Bérénice, in E., p. 556-557 et 559. 
ments qui unissent les phrases de Simon et les peintures de Miró est donné par le rôle de premier plan assigné aux couleurs. Leur présence dans le texte est insistante, et non seulement l'écrivain évoque les teintes de manière directe (le bleu, le vert), mais aussi il croit nécessaire de préciser la nuance exacte : rose cyclamen, vert pomme, lilas fané, vermillon pâle, jaune soufre, rose crevette... Même le fait que Miró, ce grand expérimentateur de techniques et de matériaux, ait utilisé du jute en tant que support pour certaines de ses peintures, trouve sa correspondance dans quelques passages du texte simonien, dans lesquels l'on parle d'une "toile de sac ", d'un " hamac fait de vieux sacs " et encore de " sacs empilés sur les quais " ${ }^{15}$.

Ceci nous ramène à la question du titre, qui a changé à plusieurs reprises. En effet, quand bien des années après l'éditeur Jérôme Lindon propose à Simon de republier Femmes en tant que livre autonome, sans les illustrations de Miró, le titre initial n'est plus utilisable, car entre-temps il a été choisi par Philippe Sollers pour l'un de ses romans ${ }^{16}$. Simon, donc, pense à une autre formule, qui est lyrique mais un petit peu prolixe : La Lumière orange de la lampe derrière la toile de sac. Cette idée lui est suggérée par l'un des tableaux reproduits dans le livre qu'il a conçu avec la collaboration de Miró : " À un certain moment, je parle d'une femme qui appelle son enfant et puis qui laisse tomber la toile qui sert de porte, comme dans bien des maisons de paysan ou de pêcheur pauvre en Espagne, et je parle d'une lumière à l'intérieur que l'on voit luire à travers la toile. J'y ai pensé parce que dans une des peintures il y a un point jaune frotté sur la toile à sac $»^{17}$. Cependant, le choix finit par tomber sur un titre qui est encore différent : «À un endroit, il est parlé des étoiles qui se reflètent dans les remous des vagues autour des pêcheurs et de la Chevelure de Bérénice. Ce nom de constellation (ou, si vous préférez, cette constellation de mots) m'a semblé beau ${ }^{18}$. Et celle-ci

15. Voir ibid., p. 556-557.

16. Ph. Sollers, Femmes, Paris, Gallimard, 1983.

17. C. Simon, dans l'entretien avec Claude DuVerlie, p. 51-52 et in E., p. 1366. Voir La Chevelure de Bérénice, in E., p. 556.

18. C. Simon, in Claire Paulhan, "Claude Simon : 'J'ai essayé la peinture, la révolution, puis l'écriture' ", in Les Nouvelles littéraires, 15-21 mars 1984, p. $42-43$ et in E., p. 1371. Voir La Chevelure de Bérénice, in E., p. 556. 
est précisément la formule, particulièrement suggestive, qui figurera dans l'édition de $1984^{19}$.

\section{Le style, les lieux, les sensations}

Le texte de Simon est caractérisé par une forme stylistique inhabituelle, en étant structuré en soixante-neuf séquences verbales courtes ou très courtes, parfois même réduites à un seul mot. Chacune des séquences commence par une lettre minuscule et se termine sans le point final. À l'intérieur de chaque morceau il n'y a pas de signes de ponctuation, sauf, dans de rares cas, des parenthèses. Si l'auteur veut faire comprendre qu'il est en train d'amorcer une nouvelle phrase, il utilise parfois la majuscule, mais toujours sans la faire précéder par le point. Les singularités de style visent à suggérer deux choses : d'une part, que le texte est proche de certaines ouvres $\mathrm{du} \mathrm{XX}^{\mathrm{e}}$ siècle basées sur l'utilisation du stream of consciousness et de l'écriture expérimentale (Joyce, Faulkner, Beckett...) ; d'autre part, son caractère essentiellement poétique. En effet, Simon a affirmé que, par rapport à ses romans, La Chevelure de Bérénice est placé " tout à fait à part. Dans mon esprit, c'est plutôt un poème $"^{20}$. Il n'est pas nécessaire, cependant, de conférer à cette indication un sens technique, comme si, avec ce texte, l'auteur avait voulu écrire un poème en prose. Simon, par ailleurs, est en désaccord avec ceux qui distinguent d'une manière trop rigide la prose et la poésie, et il conseille de " lire plus attentivement Mallarmé, qui a proclamé que chaque fois qu'il y a 'effort au style', il y a versification $»^{21}$.

19. C. Simon, La Chevelure de Bérénice, Paris, Éditions de Minuit, 1984.

20. C. Simon, dans l'entretien avec Claire Paulhan, p. 42 et in E., p. 1362.

21. C. Simon, in "Claude Simon à la Fondation d'Hautvillers. Pour le dialogue des cultures " (exposé à un colloque de 1975), in Mireille Calle-Gruber (éd.), Les Triptyques de Claude Simon ou l'art du montage, Paris, Presses de la Sorbonne Nouvelle, 2008, p. 171. L'écrivain fait allusion à un célèbre passage mallarméen : « Le vers est partout dans la langue où il y a rythme, partout, excepté dans les affiches et à la quatrième page des journaux. Dans le genre appelé prose, il y a des vers, quelquefois admirables, de tous rythmes. Mais, en vérité, il n'y a pas de prose : il y a l'alphabet et puis des vers plus ou moins serrés : plus ou moins diffus. Toutes les fois qu'il y a effort au style, il y a versification " (Stéphane Mallarmé, "Sur l'évolution littéraire », 1891, in Euvres complètes, II, Paris, Gallimard, 2003, p. 698). 
L'orientation de ces pages de Simon, est beaucoup plus descriptive que narrative : nous pouvons les lire comme une séquence qui fait intervenir lieux, personnages et situations, mais la transition d'un élément à un autre est régie par un mécanisme de type associatif, celui par lequel une image, en s'appuyant sur quelque analogie, fait surgir dans l'esprit de l'auteur la suivante. Le discours est souvent mené d'une manière impersonnelle, et seulement dans des rares passages apparait, brièvement, la première personne : le plus souvent il s'agit de verbes qui montrent le narrateur en tant que simple observateur (" je la vis », « je vis », « je pus voir»", « je le vis », « je pouvais voir »), tandis que dans d'autres phrases il se réfere à une sensation physique (" je pouvais la sentir s'écoulant le long de mes membres", « je pouvais toujours sentir cette odeur ») ou à un souvenir d'enfance ("dans mon esprit d'enfant il me semblait») ${ }^{22}$.

Les scènes se déroulent principalement sur la plage : une femme est assise au bord de la mer avec une petite fille, trois jeunes amies se promènent amenant avec elles un chien, des pêcheurs jouent au ballon ou dorment près de leur bateau, puis, la nuit, le poussent dans l'eau, ou de l'eau vers le rivage. Mais les références à des contextes différents ne manquent pas : une femme se montre à la porte de sa maison pour appeler ses enfants, des prostituées dans un bordel sont exposés aux regards des clients, une vieille femme égorge un poulet, des soldats et des officiers se reposent à côté d'un mur en ruine, un roi enfant est entouré de dignitaires et d'évêques, et ainsi de suite...

Le seul lieu qui soit nommé explicitement est Barcelone, ce qui s'explique non seulement parce que l'action du texte se déroule le plus souvent sur une plage catalane, mais aussi parce que Barcelone est une ville, comme nous l'avons dit, particulièrement chère à l'auteur. Il l'avait visitée dans un moment historique crucial, la guerre civile, et il y avait fait des expériences suffisamment marquantes pour l'inciter à s'y référer dans des romans écrits à différentes périodes, de 1947 à 1981 (La Corde raide, Le Sacre du printemps, Le Palace, Histoire, Les Géorgiques). À un critique espagnol spécialiste de ses œuvres, Simon a écrit :

22. Voir La Chevelure de Bérénice, in E., p. 556-557, 561-563. 
Décidément, quelque chose de profond, comme une sorte de fascination (que je ressens chaque fois que je retourne à Barcelone et qui ne tient pas seulement à la proximité de mon pays maternel et de la Catalogne 'Sud') me lie à cette ville énigmatique. Peut-être, me direzvous, s'agit-il de ce sentiment qu'éprouvent les vieillards pour les lieux de leur jeunesse? Mais je crois qu'il y a encore quelque chose d'autre ${ }^{23}$.

La Chevelure de Bérénice a plusieurs similitudes avec le plus barcelonais des romans de Simon, Le Palace, publié peu d'années auparavant et centré justement sur la guerre civile 24 . Il est vrai qu'il n'y a pas de traces directes de la guerre dans le texte bref écrit en 1965, mais on peut néanmoins y retrouver quelques allusions à cet événement historique. En particulier, en décrivant les pêcheurs endormis, Simon souligne à plusieurs reprises leur ressemblance avec des morts, et il fait référence aussi à la mise en scène de cette exécution :

les longs murs d'usines en briques jaunes mal cuites bordant les rues défoncées poussiéreuses des faubourgs et contre lesquels on les aligna pour les fusiller // allongés comme endormis mais trop immobiles les mouches sur eux non pas marchant mais en grappes noires agglutinées les pieds sales chaussés d'espadrilles aussi2 ${ }^{25}$.

L'écrivain fait allusion à l'assassinat de plusieurs militants anarchistes par les communistes catalans, en 1937. Même l'un des personnages les plus remarquables du Palace, l'Américain, ami du narrateur, termine probablement sa vie de cette manière, en devenant :

l'un de ces corps au pieds sales chaussés d'espadrilles couchés le long d'un mur de fabrique ou de ferme et qui semblent dormir là, dans l'ombre mouchetée des platanes, paisibles, aussi bien indifférents aux pastilles de soleil qui rampent sur leurs yeux qu'aux nuées de mouches, jusqu'à ce qu'approchant on se rende compte qu'ils sont alignés comme pour être comptés, d'une effroyable immobilité, et barbouillés de sang séché26.

23. C. Simon, lettre du 6 janvier 1983 à Manuel A. Tost Planet, citée par celui-ci dans son essai "Claude Simon et Barcelone. Derechef ", in Cahiers Claude Simon, 5, 2009, p. 116.

24. Le Palace (1962), in E., p. 413-550.

25. La Chevelure de Bérénice, in E., p. 557-558.

26. Le Palace, in E., p. 472-473. 
Compte tenu de l'importance de la composante sensorielle dans les textes de Simon, parmi les liens possibles avec Le Palace, il faut privilégier celui concernant les sensations olfactives qui, dans l'esprit du narrateur, sont étroitement associées à l'Espagne : c'est ainsi que les nombreuses remarques, parsemées dans le roman, sur les " tenaces relents de melon pourri, de poisson frit, de caroubes entassées sur les quais, d'huile rance ${ }^{27}$ reviennent exactement dans La Chevelure de Bérénice, où l'on retrouve "l'odeur de poisson ", " la violente odeur poivrée des caroubes ", les " douceâtres effluves d'huile rance chaude » et encore l'« odeur d'huile chaude soulevant le cœur $»^{28}$. Ce n'est pas une coïncidence si Claude Simon aimait à citer, d'une manière élogieuse, la phrase que Proust attribue au protagoniste de la Recherche: "Une véritable exaltation m’avait été communiquée, non par quelque idée importante, mais par une odeur de moisi ${ }^{29}$. Et puis il ajoutait: « De même, à ma grande honte, je dois dire que ce ne sont jamais que des choses dans le genre d'une odeur de moisi qui ont provoqué chez moi quelque exaltation. Des odeurs, des couleurs, des formes, des sensations, pas des idées ${ }^{30}$.

\section{La femme et l'érotisme}

Le titre originaire, Femmes, était certainement approprié, étant donné que, dans le texte simonien, l'on perçoit bien la fascination exercée sur le narrateur par le corps de la femme. Il faut dire, cependant, qu'il a cherché à montrer des personnages féminins de tous âges (petite enfant, jeune fille, femme mûre, vieille...) et dans des rôles différents (mère, ménagère, prostituée...). En outre, même si une forte tonalité érotique brille à travers ces pages, elle ne se manifeste pas dans la représentation directe des actes sexuels, comme il arrive dans certains romans de l'auteur, mais d'une manière plus indirecte et allusive, c'est-à-dire sur un plan qui est surtout symbolique. Il convient également de prendre en compte le fait que, chez

27. Ibid., p. 462.

28. La Chevelure de Bérénice, in E., p. 555-557.

29. M. Proust, À l'ombre des jeunes filles en fleur (1919), in $\grave{A}$ la recherche du temps perdu, Paris, Gallimard, 1999, p. 395.

30. «Claude Simon à la Fondation d'Hautvillers », cit., p. 170. 
Simon, l'érotisme n'est jamais un phénomène isolé, mais fait partie de la totalité de l'existence, et peut donc être associé à des aspects différents (et même opposés) de la vie elle-même.

Considérons, pour fournir un premier exemple, le motif de la langue, en tant qu'organe corporel. Dans ces pages, elle est liée au désir masculin, suscité par la vue de la femme, un désir qui se reflète, au niveau physiologique, non seulement dans une excitation spécifique (en deux passages du texte, on fait allusion au phallus érigé ${ }^{31}$ ), mais aussi à travers la sensation évoquée de la sécheresse des lèvres, que les personnages masculins tentent d'humecter : " passant leur langue desséchée sur leurs lèvres ils la suivirent des yeux silencieusement ", "léchant leurs lèvres desséchées de leur langue poilue ", "langue passant et repassant sur les lèvres crevassées " ${ }^{32}$. Il peut même arriver que la langue devienne un substitut du phallus, car elle peut entrer en contact avec le sexe des femmes, et c'est pour cette raison que certaines personnes pensent à la protéger (et en même temps à en intensifier l'effet) au moyen d'instruments spéciaux : " ces bizarres préservatifs que je vis plus tard à Barcelone en même temps que des gaines en caoutchouc pour la langue pourvues de languettes roses ou couleur de viande crue ${ }^{33}$. Mais ceci, toujours sur le plan symbolique, expose la langue au risque d'être coupée (on serait tenté de dire "châtrée »). L'auteur l'indique clairement, tout en déplaçant le discours au niveau des animaux. Ainsi nous trouvons dans le texte une référence aux raffinements gastronomiques de certains empereurs romains ("pâtés de langue de rossignols ou quels oiseaux rouges-gorges colibris que des esclaves leur apportaient sur des plats ciselés $\left.{ }^{34}\right)$, mais aussi un passage plus prosaïque et explicite : "cet oiseau des sables [...] marchant très vite entre les herbes comme un rat criant sans doute en faisant vibrer sa langue // vieille qui saignait

31. Voir La Chevelure de Bérénice, in E., p. 559 et 563.

32. Ibid., p. 562-563.

33. Ibid., p. 561. Simon avait déjà parlé de ces instruments dans l'un de ses romans publiés auparavant, La Corde raide, Paris, Éditions du Sagittaire, 1947, p. 39.

34. La Chevelure de Bérénice, in E., p. 561. Il est ici fait référence à Héliogabale, qui «mangeait assez souvent [...] des langues de paon et de rossignol » (Elio Lampridio, Vita di Eliogabalo, Milan, Mimesis, 1994, p. 80). 
les poulets en la leur coupant avec les mêmes ciseaux rouillés dont elle se servait pour vider les poissons $\star^{35}$.

Le titre définitif, La Chevelure de Bérénice, a le mérite d'attirer l'attention sur les cheveux féminins, qui, chez Simon comme chez Baudelaire, acquièrent une valeur érotique, d'une manière analogue à tout ce qui relève de la pilosité. On peut voir poindre cette vision sensuelle, par exemple, dans une scène apparemment innocente :

sans cesser de marcher elle rattacha son peigne, noire dans le crépuscule un moment ses deux bras levés je pus voir les touffes sombres de ses aisselles ses bras levés comme deux cornes un instant le peigne aux dents noires se détachant sur le ciel disparaissant passant deux ou trois fois dans sa chevelure puis elle le planta dedans et rabaissa les bras ${ }^{36}$.

Lucien Dällenbach, en commentant un passage d'un roman de Simon de 1969, La Bataille de Pharsale, note à juste titre la présence d'une constellation d'images qui relie l'herbe, les poils des aisselles et la toison pubienne ${ }^{37}$. Le même phénomène se retrouve dans $L a$ Chevelure de Bérénice, où l'herbe prend une forme aussi improbable que symptomatique : «longues herbes gris-vert non pas plates mais cylindriques semblables à des touffes de poils ", " les herbes gris-vert faiblement agitées par le vent dunes aux aisselles velues ${ }^{38}$.

Mais le sexe de la femme apparaît également d'une manière directe, exposé au regard désirant de l'homme : "poils collés moites dans les plis obscurs de son corps noirs embrouillés semblables à ces griffonnages d'enfants appuyant fort la mine de plomb crevant le papier se déchirant fente // rose cyclamen ", " cyclamen ou plutôt lilas fané fructus ventris tui ", « antre entre ses cuisses // broussailleux $~_{39}$. Bien sûr, dans le texte de Simon, il ne manque pas une allusion à la composante olfactive : "certaines sentent la mer les coquillages

35. La Chevelure de Bérénice, in E., p. 560. La vieille qui saigne les poulets annonce celle qui fera la même chose avec un lapin dans le roman Triptyque (1973), in E., p. 741-884.

36. Ibid., p. 562-563.

37. Voir Lucien Dällenbach, Claude Simon, Paris, Éditions du Seuil, 1988, p. 76-77.

38. La Chevelure de Bérénice, in CE., p. 554 e 559.

39. Ibid., p. 556-558. 
d'autres comme si on enfouissait son visage dans la mousse trouvant au-dessous cet âcre et noir parfum d'humus $»^{40}$. Des comparaisons similaires se retrouvent dans d'autres récits de l'auteur ; pour se borner à un seul exemple, l'on peut rappeler un passage de Leçon de choses: "Une forte odeur à la fois de coquillage et d'humus monte de l'épaisse toison noire sous les fesses polies ${ }^{41}$. Le double rapprochement entre le sexe féminin et les éléments que sont la mer et la terre n'est pas fortuit, parce que, pour Simon, la femme s'identifie, en fin de compte, avec la nature et la vie elle-même ; en effet, il a pu écrire : « la chair du monde est femelle par ce fait qu'elle est capable d'engendrer et de créer $»^{42}$.

Dällenbach a donc raison quand il parle, à propos des textes du romancier, d'un "Éros tellurique et cosmique " ${ }^{43}$. Comme preuve, il cite un passage de La Route des Flandres dans lequel l'orgasme qui conclut une étreinte conduit sans transition à la contemplation des étoiles :

le cri jaillissant sans fin de sa bouche jusqu'à ce qu'il n'y ait plus rien sourds tous les deux tombés inanimés sur le côté mes bras l'enserrant toujours se croisant sur son ventre sentant contre moi ses reins couverts de sueur les mêmes coups sourds le même bélier nous ébranlant tous deux comme un animal allant et venant cognant allant et venant violemment dans sa cage puis peu à peu je commençai à voir de nouveau, distinguer le rectangle de la fenêtre ouverte et le ciel plus clair et une étoile puis une autre et une autre encore, diamantines froides immobiles $^{44}$.

\section{Le motif sidéral}

S'il n'est pas rare pour Simon d'établir un lien entre l'amour sexuel et les étoiles (ce qui explique pourquoi un texte centré sur le corps de la femme a pu prendre en définitive pour titre le nom d'une constellation), il reste toujours possible, à ses yeux, d'inverser le mouvement, c'est-à-dire que ce soient les astres qui s'humanisent

40. Ibid., p. 560.

41. C. Simon, Leçon de choses, Paris, Éditions de Minuit, 1975, p. 155.

42. Le Vent (1957), in C., p. 75.

43. L. Dällenbach, op. cit., p. 73.

44. La Route des Flandres (1960), in E., p. 397. 
grâce à la référence aux femmes et à la sexualité. On peut lire par exemple un passage extrait d'un autre ouvrage de Simon :

La Chevelure de Bérénice est dessinée par une vingtaine d'étoiles, de magnitude 4 à 6 . [...] Dans deux groupes d'étoiles rapprochées dessinant deux triangles de grandeur à peu près égale où l'on peut lire schématiquement un visage, certains peuples de l'Antiquité croyaient pouvoir situer les positions successives occupées par la tête de la femme lorsque dans un spasme elle la rejette en arrière, se cambrant, abandonnant le gland qu'elle pressait entre ses lèvres, sa main toutefois toujours crispée sur la verge tendue $»^{45}$.

On ne trouve rien d'aussi explicite, du point de vue sexuel, dans La Chevelure de Bérénice. En effet, ici l'apparition de la constellation se produit dans un contexte différent, c'est-à-dire vient en quelque sorte éclairer la scène où les pêcheurs poussent leur bateau :

chaque soir ahanant dans la nuit marron la mer marron ahanant les étoiles qui vont et viennent sous eux arc-boutés poussant du dos les éclats du fanal sur leurs épaules luisantes de sueur sortant de la nuit épaisse à chaque poussée on peut entendre craquer les membrures l'énorme barque se profilant monstrueuse noire sur le fond des constellations le Bouvier les Chiens de Chasse l'Hydre femelle leurs jambes pataugeant dans les étoiles entrechoquées la masse obscure gémissant oscillant mais toujours désespérément inerte chaque soir les vagues marron sortant l'une après l'autre du fond de la nuit se brisant avançant lentement en lignes parallèles déferlant déroulant le tapis de reflets [...] // reprenant souffle silencieux alignés immobiles sans se détacher de lui collés par le dos à chaque vague les étoiles rapides se ruaient autour de leurs jambes les éclaboussant puis se retiraient la Chevelure de Bérénice Pégase laissant voir leurs chevilles à demi enfoncées dans le sable marron chaque soir jour après jour ${ }^{46}$.

Une scène qui, en elle-même, est humble et quotidienne acquiert, dans les pages de Simon, un air solennel et presque épique. L'écrivain met l'accent sur l'effort physique des pêcheurs aux prises avec le bateau difficile à déplacer, mais décrit aussi, d'une manière lyrique, une situation dans laquelle tout est mêlé : les ténèbres et la lumière, les corps des hommes et la coque du bateau, l'eau de mer

45. C. Simon, Les Corps conducteurs, Paris, Éditions de Minuit, 1971, p. 57-58.

46. La Chevelure de Bérénice, in E., p. 555-556. 
et les étoiles. Même les couleurs ont fusionné, de sorte qu'on peut qualifier de "marron " aussi bien la nuit et la mer que le sable. L'on retrouvera un épisode assez semblable, présenté comme un souvenir d'enfance du narrateur, dans le dernier livre simonien, Le Tramway. Dans ce cas, toutefois, les images seront à la fois plus réalistes (puisque, à propos des pêcheurs, seront donnés des détails prosaïques comme la mention de leurs "pieds noueux et poilus " ou l'allusion aux jurons qu'ils proferent) et autrement symboliques. En fait, la lumière qui vient des fanaux posés à terre, et qui illumine les pêcheurs d'en dessous, fera surgir la comparaison avec « cet on ne savait quoi de mystérieux et de sacré qui, dans les tableaux ou les illustrations du catéchisme représentant la Nativité, transforme et magnifie ceux des bergers accourus éclairés de même par la lumière émanant du berceau ", et peu après les poissons qui remplissent la poche du filet donneront " à la scène une dimension en quelque sorte biblique et quelque peu féerique comme la Pêche miraculeuse ou la Multiplication des pains $"^{47}$. Dans La Chevelure de Bérénice, Simon s'abstient soigneusement d'établir des comparaisons de ce genre. Si l'on voulait parler du sacré en référence au texte de 1965, il faudrait le faire par rapport à l'attitude, plus païenne que chrétienne, qui est décelable dans tous les livres de l'auteur. Elle peut être résumée par la phrase de $\mathrm{La}$ Corde raide qui pose « que l'on n'arriv[e] jamais à se rassasier de cette somptueuse magnificence du monde, pourvu que l'on parv[ienne] à en être conscient ${ }^{48}$.

L'ancienne légende de la Chevelure de Bérénice est donc en parfait accord avec la poétique de Simon, qui tend à établir un rapport entre les petits éléments physiques et concrets, auxquels est liée la vie de l'homme, et les plus vastes cycles de la nature et de l'Histoire. Il n'y a rien d'insolite, pour lui, à imaginer la métamorphose d'une natte de cheveux féminins en constellation, ou à décrire la descente des étoiles, fut-ce à travers leur reflet, à la surface de la mer, au moment où elles se trouvent à distance immédiate des jambes des pêcheurs au travail. L'image de la voûte céleste étoilée qui se reflète dans la mer peut paraître surprenante, mais après tout elle

47. Cf. C. Simon, Le Tramway, Paris, Éditions de Minuit, 2001, p. 50-51.

48. La Corde raide, cit., p. 64. 
donne raison aux critiques littéraires qui ont retrouvé en Simon un " esprit baroque " ${ }^{49}$. Ce n'est pas un hasard si l'un des principaux représentants de la poésie baroque européenne, Giambattista Marino, a décrit dans l'un de ses poèmes un échange similaire entre le ciel et l'eau de mer :
Regarde la mer, Craton, en ce moment où Entre ses rivages les vagues dorment, le vent se tait Et la Nuit, dans le ciel, déploie sa riche robe Bleu et brune, ornée de cent et cent joyaux. $[\ldots]$
Vois comme là-dessus et ici-bas s'en vont en jouant, Avec des étincelles ardentes et claires, les étoiles Changées en poissons, les poissons en étoiles. Tellement pur le beau fond de la mer se montre à nous, Que, parmi tant d'éclairs et de flammèches, tu diras : - Voici que la mer est changée en ciel limpide. $-{ }^{50}$.

Pour Simon, le cosmos est unitaire, mais ce qui lui donne le mouvement n'est pas une divine providence, mais une chose bien plus terrestre, et pourtant efficace : l'impulsion de la vie qui veut continuer, le conatus de Spinoza, " la pulsion à partir de laquelle chaque chose est amenée à persévérer dans son être ». Cette pulsion, "quand elle se rapporte en même temps à l'esprit et au corps est appelée appétit » ou "cupidité " ${ }^{51}$. Il est ici plutôt question de désir, y compris de désir sexuel, qui, bien que contrarié par mille obstacles, et enfin annulé par la mort, tend cependant à prolonger l'existence de l'homme aussi longtemps que possible. Et de ce désir un texte comme La Chevelure de Bérénice est une célébration lyrique particulièrement puissante.

49. C'est une formule que l'on lit dans le livre de Simonetta Micale, Il fotogramma e la frase. Incontri di fotografia e scrittura nell'opera di Claude Simon, Naples, Edizioni Scientifiche Italiane, 2005, p. 63.

50. G. Marino, "Tranquillità notturna ", in La Lira (1614), in Poesie varie, Bari, Laterza, 1913, p. 99.

51. Baruch Spinoza, Etica, III, in Opere, Milan, Mondadori, 2007, p. 905-907. 